\title{
Metabolic Syndrome and Breast Cancer: Is There a Link?
}

\author{
Dagmar Hauner Hans Hauner
}

Else Kröner-Fresenius-Center for Nutritional Medicine, Klinikum rechts der Isar, Technical University Munich, Germany

\section{Keywords}

Abdominal obesity - Metabolic syndrome .

Breast cancer - Triple-negative breast cancer (TNBC)

\section{Summary}

Epidemiological data suggest a close link between obesity and breast cancer, the most frequently occurring cancer in women. The metabolic syndrome is typically associated with abdominal obesity and comprises disturbances in glucose and/or lipid metabolism and/or hypertension. Recent studies have established a specific association between the metabolic syndrome - as well as its components - and breast cancer, indicating both an increased risk of developing breast cancer and a poorer prognosis. In premenopausal women, obesity might have a protective effect only on receptor-positive tumors, whereas a positive association was observed between obesity/abdominal obesity and an increased risk of triple-negative breast cancer (TNBC). Overall survival and disease-free survival were reported to be significantly shorter in premenopausal obese women with TNBC compared to non-obese women, but these results are still inconsistent and need further research. The metabolic syndrome is characterized by a state of insulin resistance/hyperinsulinemia and subacute chronic inflammation, with both conditions offering a plausible mechanistic link towards breast cancer. Thus, in addition to their increased risk of cardiovascular morbidity and mortality, women with this syndrome represent a group at elevated risk of developing breast cancer and with poorer prognosis.

\section{Introduction}

Breast cancer is the most common malignancy in women worldwide. The lifetime risk of a woman being diagnosed with breast cancer is approximately $12 \%$ in the Western countries [1]. It is interesting to note that there is substantial variation in the breast cancer risk across countries, probably due to differences in lifestyle and environmental factors. Understanding how modifiable lifestyle risk factors affect breast cancer incidence may have important implications for the prevention and management of this malignancy, especially in countries with a high disease burden [1].

Over the last decade, the associations between obesity, physical activity, diet and the risk of developing breast cancer have been extensively studied and are now well established $[2,3]$. Recently, it turned out that obesity is probably the most powerful modifiable risk factor for the incidence and prognosis of breast cancer [4-6]. Obesity with preferential intraabdominal fat accumulation ('abdominal obesity') is well known to be a potent promoter of cardiovascular risk factors including glucose and lipid disorders and elevated blood pressure. This cluster of risk factors along with abdominal obesity is commonly referred to as metabolic syndrome [7].

\section{Definition and Prevalence of the Metabolic Syndrome}

The current definition of the metabolic syndrome originates from 2001 [8], and after intensive discussion among expert groups, a unified definition has been recently established [9] (table 1). In Caucasian populations, a waist circumference threshold of $102 \mathrm{~cm}$ in men and $88 \mathrm{~cm}$ in women is usually preferred as a measure of abdominal obesity. When 3 of the 5 criteria are present, the diagnosis of metabolic syndrome is fulfilled $[8,9]$.

\section{KARGER \\ Fax +497614520714 \\ Information@Karger.com}

www.karger.com (c) 2014 S. Karger GmbH, Freiburg

$1661-3791 / 14 / 0094-0277 \$ 39.50 / 0$

Accessible online at:

www.karger.com/brc 
Table 1. Criteria

for the clinical diagnosis of the metabolic syndrome, according to Alberti et al. [9]

\begin{tabular}{ll}
\hline Measure & Categorical cut points \\
\hline $\begin{array}{l}\text { Elevated waist circumference } \\
\text { Elevated triglycerides (drug treatment for elevated triglycerides } \\
\quad \text { is an alternate indicator) }\end{array}$ & $\begin{array}{l}\text { population- and country-specific definitions } \\
\text { Reduced HDL-cholesterol (drug treatment for reduced HDL- } \\
\text { cholesterol is an alternate indicator) }\end{array}$ \\
$\begin{array}{l}\text { Elevated blood pressure (antihypertensive drug treatment in a } \\
\text { patient with a history of hypertension is an alternate indicator) }\end{array}$ & $<40 \mathrm{mg} / \mathrm{dl}(1.0 \mathrm{mmol} / \mathrm{l})$ in men \\
$\begin{array}{l}\text { Elevated fasting glucose (drug treatment for elevated glucose is } \\
\text { an alternate indicator) }\end{array}$ & $\geq 100 \mathrm{mg} / \mathrm{dl}$
\end{tabular}

In Western countries, the prevalence of the metabolic syndrome was reported to be between 20 and $25 \%$ in the adult population. It is noteworthy that there is a marked increase in prevalence by age, leading to rates of $40-45 \%$ in those aged 50 years or older $[10,11]$. The presence of metabolic syndrome indicates a 2-3-fold increased risk of cardiovascular morbidity and mortality in those fulfilling the criteria compared to those without the syndrome [7].

\section{Metabolic Syndrome and Breast Cancer}

Only recently, several cohort studies suggested an association between the metabolic syndrome and breast cancer incidence. In a systematic review and meta-analysis, Esposito et al. [12] identified 9 studies on the association between the metabolic syndrome and the risk of postmenopausal breast cancer, including a total number of 6,417 incident cancer cases. The results indicate that the presence of the metabolic syndrome is associated with a $52 \%$ increase in breast cancer risk $(\mathrm{p}<0.001)$.

An important question arising from this observation is which components of the metabolic syndrome may be responsible for this association. The above-cited study analyzed the 5 components of the metabolic syndrome separately; the risk estimates for postmenopausal breast cancer were $1.12(\mathrm{p}=0.068)$ for an elevated body mass index (BMI)/waist circumference, $1.19(\mathrm{p}=0.005)$ for hyperglycemia (higher fasting glucose or diabetes), $1.13(\mathrm{p}=0.027)$ for elevated blood pressure, 1.08 $(\mathrm{p}=0.248)$ for elevated triglycerides, and $1.39(\mathrm{p}=0.008)$ for low levels of high-density lipoprotein (HDL) cholesterol compared to $1.52(\mathrm{p}<0.001)$ for the full syndrome [12].

Although there is limited knowledge as to whether the presence of the metabolic syndrome affects the prognosis of women with breast cancer, some recent studies with mostly small sample sizes showed a reduced survival. In a large cohort of 287,320 women, the presence of the metabolic syndrome resulted in an increased breast cancer mortality risk for women aged $\geq 60$ years (relative risk (RR) 1.23 ; $95 \%$ confidence interval (CI) 1.04-1.45), which was not confirmed for younger women [13]. Thus, there is growing evidence that the metabolic syndrome is associated with both an increased incidence of breast cancer and a worsening of its prognosis.

\section{Obesity and Breast Cancer}

There is now overwhelming evidence indicating that an excess of body fat represents an independent risk factor for both an increased incidence and a poorer prognosis of breast cancer [2, 4-6]. This relationship holds particularly true for the risk of postmenopausal breast cancer, whereas obesity was previously reported to be protective against the development of premenopausal breast cancer [2]. However, it turned out that obesity also impairs the survival in premenopausal women with newly diagnosed breast cancer [4-6]. In the meta-analysis of Protani et al. [4], a significantly poorer prognosis was found among obese compared to non-obese women with breast cancer, which was similar for overall survival (hazard ratio (HR) 1.33; 95\% CI 1.21-1.47) and breast cancer-specific survival (HR 1.33; 95\% CI 1.19-1.50). Interestingly, this positive association was also observed in premenopausal women [4], as confirmed by a current metaanalysis [6]. A recent paper also indicated obesity as an unfavorable independent prognostic factor for the development of distant metastases and death [14].

\section{Obesity/Metabolic Syndrome and Breast Cancer Subtypes}

Studies in postmenopausal women have observed a strong association between obesity and the risk of estrogen receptor $(\mathrm{ER})+$ /progesterone receptor $(\mathrm{PR})+$ breast cancer, but no association for ER+/PR- or ER-/PR- breast cancer [15]. A pooled analysis revealed that, among women $<50$ years of age, obesity was associated more frequently with ER-/PR- tumors than with $\mathrm{ER}+\mathrm{PR}+$ tumors and, by including human epidermal growth factor receptor 2 (HER2) receptors, obesity was confined to triple-negative tumors (TNBC) (triple-negative vs. ER+/HER2 - or PR+/HER2- tumors: odds ratio (OR) 1.80; $95 \%$ CI 1.42-2.29; $\mathrm{p}=0.000006$ ) [16]. Obviously, obesity in younger women has a protective effect only in case of receptorpositive tumors [16]. Another recent meta-analysis confirmed a positive association between obesity and TNBC in premenopausal but not in postmenopausal women, although in the latter group hormone therapy was not taken into account [17]. 
Only a few studies examined the relationship between metabolic syndrome and hormone receptor status in women with breast cancer. In 1 large cohort study, no significant association was found in postmenopausal women when adjusting for BMI [18]. Data on the relationship between the metabolic syndrome and TNBC are limited, concerning mostly primary prevention and single components of the metabolic syndrome [19]. A positive association between an elevated waist-hip ratio (WHR) and an increased risk of TNBC was seen in some studies [19, 20].

As to prognosis, a recent meta-analysis found a higher BMI to be consistently related to lower overall survival and breast cancer-specific survival, but no significant differences were seen concerning the receptor status [6]. Overall survival and disease-free survival were reported to be significantly shorter in premenopausal obese women with TNBC compared to non-obese women [21]. However, in another study, obesity was not associated with decreased survival in patients with TNBC [22], indicating the need for more and larger studies to clarify this relationship.

\section{Diabetes and Breast Cancer}

Type 2 diabetes is closely associated with abdominal obesity and is a frequent component of the metabolic syndrome. In the US Cancer Prevention Study II enrolling 588,321 women free of cancer, diabetes was significantly associated with increased breast cancer morbidity in women after 16 years of follow-up (RR 1.27; 95\% CI 1.11-1.45) [23]. This association was not explained by BMI or other potential confounding factors [23]. A recent meta-analysis of these studies summarized that patients with diabetes and breast cancer have a significantly higher all-cause mortality compared to patients without diabetes (HR 1.49; 95\% CI 1.35-1.65). In addition, patients with pre-existing diabetes also tended to present at later stages and to receive altered treatment regimens [24]. In addition, clinical data suggest that the diabetic state may promote a more aggressive cancer phenotype and/ or a poorer response to treatment. Thus, diabetes increases the risk of breast cancer and worsens its prognosis.

On the other hand, a recent retrospective study showed that women with diabetes who were treated with metformin had a significantly lower risk of developing breast cancer than women without metformin use (OR 0.44; 95\% CI 0.24 0.82) [25]. In a study from Denmark, even short-term treatment with metformin reduced the risk of breast cancer in a multivariate analysis [26]. Thus, not all [27] but the majority of clinical data suggests a beneficial effect of metformin. In contrast, some concern was expressed regarding the impact of long-term insulin treatment, in particular with insulin glargine, on the breast cancer risk. In a recent meta-analysis, the results of studies including 448,928 subjects and 19,128 cancer patients were analyzed: Glargine did not increase the
OR of breast cancer (OR 0.99; 95\% CI 0.68-1.46), arguing against a specific adverse effect. However, there is agreement that more clinical trials are needed due to the low quality of many previous studies [28].

\section{Potential Mechanisms Underlying the Association between Metabolic Syndrome/Abdominal Obesity and Chronic Diseases}

The mechanisms underlying the pathophysiology of the metabolic syndrome and its association with breast cancer and cardiovascular disease have been only partially elucidated. A common feature that probably links all components of the metabolic syndrome and characterizing abdominal obesity is insulin resistance, which can be defined as an impaired response to insulin leading to a compensatory increased insulin secretion and hyperinsulinemia. Another characteristic of the metabolic syndrome/abdominal obesity is subacute chronic inflammation caused by an accumulation of immune cells in adipose tissue and an impaired secretion of 'adipokines' including a variety of pro-inflammatory cytokines, further promoting a state of systemic insulin resistance and chronic inflammation. There is growing evidence that the combination of insulin resistance, subacute chronic inflammation, and increased visceral fat mass may represent a 'common soil' not only for metabolic and atherosclerotic diseases but also for many other diseases including some types of cancer. However, the clinical consequences of the metabolic syndrome are highly variable, probably depending on the respective individual genetic background and modifying environmental factors.

\section{Pathophysiology of the Association between Metabolic Syndrome and Breast Cancer}

The hypothesis of a 'common soil' underlying the metabolic syndrome, originally introduced by Stern [29] and characterized by insulin resistance and chronic inflammation, may be also relevant in the pathophysiology of breast cancer and its progression. There are more and more hints that the same mechanisms are operating in both cases (fig. 1). Some pathophysiological features are discussed in the following.

Insulin resistance is frequently observed in obesity and in subjects on a Western diet and/or those lacking physical activity. Insulin is known to have mitogenic, antiapoptotic, and angiogenic properties. These biological actions of insulin may explain why elevated levels of fasting insulin have been implicated in cancer progression and mortality [30]. In addition, insulin was found to stimulate the synthesis of insulinlike growth factor 1 (IGF-1) and to weakly bind to the IGF-1 receptor. In general, insulin-IGF-1 signaling on tumor cells is up-regulated in obesity and results in an activation of the on- 


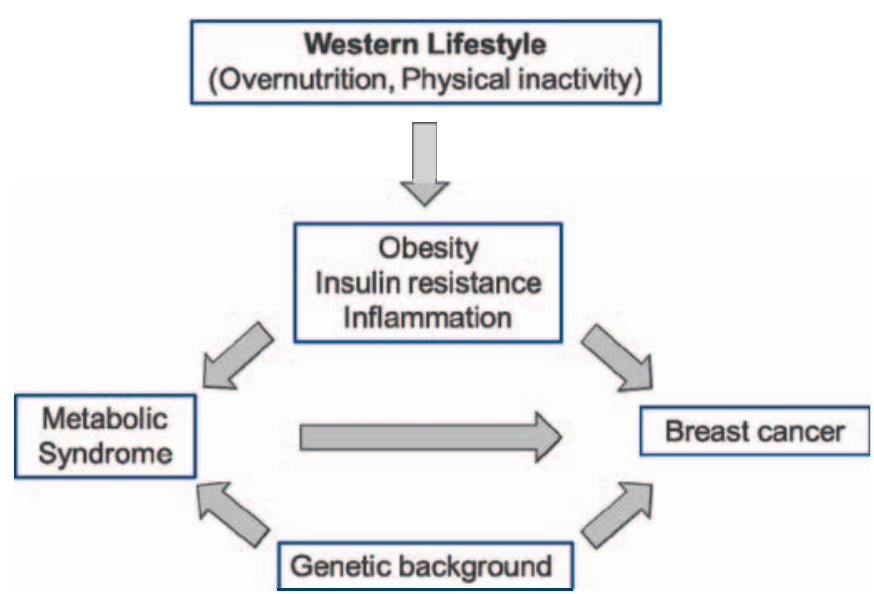

Fig. 1. Simplified scheme of the relationship between metabolic syndrome and breast cancer.

cogenic Ras-MAPK and PI3K-Akt pathways (MAPK = mitogen-activated protein kinase, PI3K = phosphoinositide 3-kinase). Further, the Akt pathway activates mammalian target of rapamycin (mTOR), which is known to stimulate tumor cell growth [31].

Chronic low-grade inflammation is another feature of the metabolic syndrome and abdominal obesity. Adipose tissue is an important production site for pro- and anti-inflammatory proteins, and an expansion of adipose tissue mainly due to fat cell hypertrophy results in an increased release of proinflammatory proteins [32]. In addition, this local inflammation is also characterized by the invasion and accumulation of activated immune cells (e.g., lymphocytes and monocytes/ macrophages) and a decreased production of anti-inflammatory proteins such as adiponectin [31].

The pro-inflammatory factors released from adipose tissue, including tumor necrosis factor- $\alpha$ (TNF- $\alpha)$, interleukin (IL)-1 $\beta$, IL-6, IL-8, and monocyte chemoattractant protein-1 (MCP-1), among others, may exert mitogenic, angiogenic, and antiapoptotic actions and have been implicated in tumor progression. Another interesting product released from adipose tissue is the fat cell hormone leptin, which is circulating at high concentrations in the blood of obese people and has been reported to have angiogenic properties [31, 33].

Adipose tissue is a major component of the tumor microenvironment in breast tissue and there are interactions between cancer cells and adjacent adipocytes leading to socalled cancer-associated adipocytes (CAA), which by their specific secretory profile support tumor growth. These adipocytes in direct contact to breast cancer cells show delipidation and loss of terminal differentiation markers, as well as an increased expression of pro-inflammatory cytokines including IL-6 and plasminogen activator inhibitor type 1 (PAI-1), suggesting an intensive cross-talk between adipocytes and cancer cells. The increased lipolytic activity may serve to supply energy to the growing cancer cells [34].
In postmenopausal women, adipose tissue becomes the major production site for estrogens via the enzyme aromatase which is expressed by the stromal cell fraction [35]. As obese postmenopausal women usually exhibit elevated estrogen levels, they are at risk of developing estrogen-dependent breast cancer. Furthermore, adipose tissue estrogen production is partially explained by an increased local release of TNF- $\alpha$, IL-6, and prostaglandin E2 [35], which can also contribute to the development of breast cancer.

\section{Perspectives and Practical Consequences for the Prevention and Treatment of Breast Cancer}

Reducing adipose tissue and, in particular, the visceral fat mass is considered to be the most effective way to improve the clinical features of the metabolic syndrome. Therefore, weight loss in combination with regular physical activity appears to be the most powerful intervention to reduce insulin resistance and inflammation. But, to date, only limited information is available on the potential benefit of weight loss for both the incidence and progression of breast cancer. The current evidence for recommending weight loss to achieve these goals has been recently reviewed [36]. The general message from this analysis was that intentional weight reduction improves surrogate markers of tumor risk and progression, e.g. increased adiponectin levels and decreased free-estrogen concentrations and C-reactive protein (CRP) levels. At present, there are 2 large ongoing prospective studies on the effect of weight loss on survival [37] and comorbidities $[37,38]$ in breast cancer patients.

Another potential option for the prevention of breast cancer and the improvement of its prognosis is adherence to a Mediterranean diet. Some recent cohort studies observed a risk-lowering association [39, 40], and the ongoing DIANA-5 trial investigates the effects of a Mediterranean macrobiotic lifestyle on breast cancer prognosis [41].

Concerning pharmacological strategies, the only relevant choice at present seems to be metformin, a long-established blood glucose-lowering agent for the therapy of type 2 diabetes. Treatment of early-stage breast cancer patients with metformin was reported to reduce plasma insulin and, moderately, body weight [42]. Metformin is known to exert several insulin-independent effects such as activation of AMPactivated protein kinase (AMPK) with subsequent inhibition of the mTOR signaling pathway. Therefore, it appears plausible to pursue the hypothesis that metformin may be effective in breast cancer prevention and treatment, which is investigated in ongoing clinical trials [43].

In conclusion, metabolic syndrome and breast cancer share several common pathophysiological features such as insulin resistance and low-grade chronic inflammation and both are promoted by obesity and other unfavorable lifestyle factors. This observation may help to understand the associ- 
ation between both entities, but may also be valuable for the development of novel strategies to prevent breast cancer and to improve its prognosis. Furthermore, in premenopausal women, obesity/metabolic syndrome may promote the development of TNBC, which is a novel finding and may require additional studies.

\section{Disclosure Statement}

Both authors declare that there is no conflict of interest.

\section{References}

1 Soerjomataram I, Lortet-Tieulent J, Parkin DM, et al.: Global burden of cancer. Lancet 2012;380: 1840-1850.

2 World Cancer Research Fund/American Institute for Cancer Research: Food, Nutrition, Physical Activity, and the Prevention of Cancer: A Global Perspective. Washington, DC, American Institute for Cancer Research (AICR), 2007.

3 Friedenreich CM: Physical activity and breast cancer: review of the epidemiological evidence and biological mechanisms. Recent Results Cancer Res 2011;188:125-139.

4 Protani M, Coory M, Martin JM: Effect of obesity on survival of women with breast cancer: systematic review and meta-analysis. Breast Cancer Res Treat 2010;123:627-635.

5 Hauner D, Janni W, Rack B, Hauner H: The effect of overweight and nutrition on prognosis of breast cancer. Dtsch Arztebl Int 2011;108:795-801.

6 Chan DSM, Vieira AR, Aune D, et al.: Body mass index and survival in women with breast cancer systematic literature review and meta-analysis of 82 follow-up studies. Ann Oncol 2014; [Epub ahead of print], doi: 10,1093/annonc/mdn042.

7 Eckel RH, Grundy SM, Zimmet PZ: The metabolic syndrome. Lancet 2005;365:1415-1428.

$\checkmark 8$ Expert Panel on Detection, Evaluation, and Treatment of High Blood Cholesterol in Adults: Executive summary of the third report of the National Cholesterol Education Program (NCEP) expert panel on detection, evaluation and treatment of high blood cholesterol in adults (Adult Treatment Panel III). JAMA 2001;285:2486-2497.

9 Alberti KGMM, Eckel RH, Grundy SM, et al.: Harmonizing the metabolic syndrome. A joint interim statement of the International Diabetes Federation Task Force on Epidemiology and Prevention, National Heart, Lung, and Blood Institute, American Heart Association, World Heart Federation, International Atherosclerosis Society, and International Association for the Study of Obesity. Circulation 2009;120:1640-1645.

10 Ford ES, Giles WH, Dietz WH: Prevalence of the metabolic syndrome among US adults: findings from the third National Health and Nutrition Examination Survey. JAMA 2002;287:356-359.

11 Moebus S, Hanisch J, Bramlage P, et al.: Regional differences in the prevalence of the metabolic syndrome in primary care practices in Germany. Dtsch Arztebl Int 2008;105:207-213.

$\checkmark 12$ Esposito K, Chiodini P, Capuano A, et al.: Metabolic syndrome and risk of postmenopausal breast cancer: a systematic review and meta-analysis. Menopause 2013;20:1301-1309.

13 Bjørge T, Lukanova A, Jonsson H, et al.: Metabolic syndrome and breast cancer in the me-can (metabolic syndrome and cancer) project. Cancer Epidemiol Biomarkers Prev 2010;19:1737-1745.
Ewertz M, Jensen M-B, Gunnarsdottir KA, et al.: Effect of obesity on prognosis after early-stage breast cancer. J Clin Oncol 2011;29:25-31.

15 Ligibel JA, Strickler HD: Obesity and its impact on breast cancer: tumor incidence, recurrence, survival, and possible interventions. Am Soc Clin Oncol Educ Book 2013;52-59.

16 Yang XR, Chang-Claude J, Goode EL, et al.: Associations of breast cancer risk factors with tumor subtypes: a pooled analysis from the Breast Cancer Association Consortium studies. J Natl Cancer Inst 2011;103:250-263.

17 Pierobon M, Frankenfeld CL: Obesity as a risk factor for triple-negative breast cancers: a systematic review and meta-analysis. Breast Cancer Res Treat 2013;137:307-314.

18 Reeves KW, McLaughlin V, Fredman L, et al.: Components of metabolic syndrome and risk of breast cancer by prognostic features in the study of osteoporotic fractures cohort. Cancer Causes Control 2012;23:1241-1251.

19 Davis AA, Kaklamani VG: Metabolic syndrome and triple-negative breast cancer: a new paradigm. Int J Breast Cancer 2012;2012:809291.

20 Vona-Davis L, Rose DP, Hazard H, et al.: Triplenegative breast cancer and obesity in a rural Appalachian population. Cancer Epidemiol Biomarker Prev 2008;17:3319-3324.

21 Turkoz FP, Solak M, Petekkaya I, et al.: The prognostic impact of obesity on molecular subtypes of breast cancer in premenopausal women. J BUON 2013;18:335-341.

22 Mowad R, Chu QD, Li BD, et al.: Does obesity have an effect on outcomes in triple-negative breast cancer? J Surg Res 2013;184:253s-259s.

23 Coughlin SS, Calle EE, Teras LR, et al.: Diabetes mellitus as a predictor of cancer mortality in a large cohort of US adults. Am J Epidemiol 2004; 159:1160-1167.

24 Peairs KS, Barone BB, Snyder CF, et al.: Diabetes mellitus and breast cancer outcomes: A systematic review and metaanalysis. J Clin Oncol 2011;29:40-46.

25 Bodmer M, Meier C, Krayenbuhl S, et al.: Longterm metformin use is associated with decreased risk of breast cancer. Diabetes Care 2010;33:1304-1308.

26 Bosco JL, Antonsen S, Sorensen HT, et al.: Metformin and incident breast cancer among diabetic women: a population-based case-control study in Denmark. Cancer Epidemiol Biomarkers Prev 2011;20:101-111.

27 Lega IC, Austin PC, Gruneir A, et al.: Association between metformin therapy and mortality after breast cancer: a population-based study. Diabetes Care 2013;36:3018-3026.

28 Tang X, Yang L, He Z, Liu J: Insulin glargine and cancer risk in patients with diabetes: a meta-analysis. PLoS One 2013;7:e51814.
29 Stern MP: Diabetes and cardiovascular disease: the 'common soil' hypothesis. Diabetes 1995;44:369-374.

30 Goodwin PJ, Ennis M, Pritchard KI, et al.: Fasting insulin and outcome in early-stage breast cancer: results of a prospective cohort study. J Clin Oncol 2002;20:42-51.

31 Khandekar MJ, Cohen P, Spiegelman BM: Molecular mechanisms of cancer development in obesity. Nat Rev Cancer 2011;11:886-895.

32 Skurk T, Alberti-Huber C, Herder C, Hauner H: Relationship between adipocyte size and adipokine expression and secretion in human adipose tissue. $\mathrm{J}$ Clin Endocrinol Metab 2007;92:1023-1033.

33 Park J, Euhus DM, Scherer PE: Paracrine and endocrine effects of adipose tissue on cancer development and progression. Endocr Rev 2011;32:550-570.

34 Nieman KM, Romero IL, Van Houten B, Lengyel E: Adipose tissue and adipocytes support tumorigenesis and metastasis. Biochim Biophys Acta 2013;1831:1533-1541.

35 Simpson ER, Brown KA: Obesity and breast cancer: role of inflammation and aromatase. J Mol Endocrinol 2013;51:T51-T59.

36 Byers T, Sedjo RL: Does intentional weight loss reduce cancer risk? Diabetes Obes Metab 2011; 32:550-570.

37 Rack B, Andergassen U, Neugebauer J, et al.: The German SUCCESS C study - the first European lifestyle study on breast cancer. Breast Care 2010; 5:395-400.

38 Rock CL, Byers T, Colditz GA, et al.: Reducing breast cancer recurrence with weight loss, a vanguard trial: the Exercise and Nutrition to Enhance Recovery and Good Health for You (ENERGY) trial. Contemp Clin Trials 2013;34:288-295.

39 Cottet V, Touvier M, Fournier A, et al.: Postmenopausal breast cancer risk and dietary patterns in the E3N-EPIC prospective cohort study. Am J Epidemiol 2009;170:1257-1267.

40 Trichopoulou A, Bamia C, Lagiou P, Trichopoulos D: Conformity to traditional Mediterranean diet and breast cancer risk in the Greek EPIC cohort. Am J Clin Nutr 2010;92:620-625.

41 Villarini A, Pasanisi P, Traina A, et al.: Lifestyle and breast cancer recurrences: the DIANA-5 trial. Tumori 2012;98:1-18.

42 Goodwin PJ, Pritchard KI, Ennis M, et al.: Insulin-lowering effects of metformin in women with early breast cancer. Clin Breast Cancer 2008; 8:501-505.

43 Goodwin PJ, Stambolic V: Obesity and insulin resistance in breast cancer - chemoprevention strategies with a focus on metformin. Breast 2011;20 (suppl 3):S31-S35. 